\title{
Heterogeneous Buffer Size Impact on UDP Performance for Real-Time Video Streaming Application
}

\author{
Sarfraz Ahmed Soomro, M. Mujtaba Shaikh, Nasreen Nizamani, Ehsan Ali Buriro, Khalil M. Zuhaib \\ Department of Electronic Engineering, \\ QUCEST, Larkana, Sindh, Pakistan
}

\begin{abstract}
Communication specifically in real-time (RTC) is a terminology which insinuates any live media transmission that occurs inside time limits. In this paper, heterogeneous buffer sizes in random are utilized on different routers and for different ranges to examine their effect on the performance of network for user datagram protocol's (UDP) video streaming application. It appeared through numerical results that packet switches heterogeneous buffer sizes as a rule influence the general performance of the network. By thinking about bigger range of buffer sizes, throughput improves but End-to-End delay also increases which is customarily not commendable for RTC applications. On the contrary, throughput decreases on account of considering low range of buffer sizes; however, End-to-End delay additionally diminishes. In this manner, the middle of the road scope of buffer sizes range from 30 to 20 , recommended for ideal throughput and an adequate lower End-to-End delay.
\end{abstract}

Keywords-Real-time communication; buffer size; user datagram protocol; video streaming

\section{INTRODUCTION}

Video streaming is an important application of real-time communication (RTC) which is a live transmission of anything. Any important factor for an RTC application is its timeliness. Buffer is a type of memory usually used for controlling the congestion which occurs in networks by holding the packets of data for a certain period and such period is known as threshold. The threshold is very important factor in buffers, used to hold the data. If the time exceeds (delay) the specified threshold limit, the data would be lost. As timeliness is an important parameter for RTC applications like video streaming, the data packets must reach the destination in due course of time otherwise the data will be considered as lost [1]. The delay usually occurs due to the transmission, processing, queueing, and propagation which is usually called as End-toEnd latency or delay. For the superior functionality of the network, there should be high throughput and low latency. User Datagram Protocol (UDP) is an important protocol suite for internet. It has been investigated by many researchers that UDP is a paramount protocol for RTC applications like live video streaming. In this research, the main focus is to investigate the influence of heterogeneous size of buffer on the performance of a video streaming application in real-time. Many people have worked on buffer size of the routers. Subsequently, it was proposed in [2] that as a rule-of-thumb, buffer size cannot be used. In [3], it was exhibited that traffic's round-trip times and loss synchronization are used to find out the size of buffer. In [4], real-time video streaming application was used to assess the networks for their buffer size. It was concluded that real-time video streaming losses are due to high End-to-End delay and jitter. It additionally demonstrated that TCP activity of traffic and also non-TCP type may hurtfully affect each other. In [5], the buffer size of the routers was changed and showed the results of throughput and packet loss of TCP and UDP for both live and non-live applications under two essential overseeing procedures for a waiting queue, Drop Tail and Random Early Detection (RED). The obtained numerical results proved that efficiency and throughput regarding TCP application enhances with a development in size of buffer for RED and packet loss is reducing. In [6], authors considered a heterogeneous wireless network for applications of multimedia type and attempted to choose UDP a magnificent component for transport layer and all other layers to bypass TCP issues. Most of the researchers have not considered the heterogeneous buffer size of the routers. Apart from the others work, in this paper, End-to-End latency or delay and throughput have been examined for heterogeneous buffer sizes of the network routers under live video streaming application.

In rest of the paper under Section II, the proposed network model is discussed. Section III describes the performance indicators which includes average latency, throughput, and buffer size of the router. Numerical results of the paper are explained in Section IV. Finally, the conclusion is presented in Section V.

\section{NETWORK MODEL}

The model of proposed network is appeared in Fig. 1 where OMNET++ using NED (Network Descriptive) language ${ }^{1}$ has been utilized to build up the network system. This network comprises of nine (09) routers and twenty-four (24) hosts to analyze the throughput, a benchmark metric and latency or End-to-End delay for the video streaming type traffic.

Video streaming is one of the innovation, utilized for sound and video broadcasting in real-time over the web. In live time, live substance, for instance, a political level-headed discussion, any games, or a talk show can be transmitted [7] for which the record is played out while parts of the document are being perceived and decoded just in this mode as there is no necessity

\footnotetext{
${ }^{1}$ http://inet.omnetpp.org/doc/INET/inet-manual-draft.pdf

https://github.com/inetmanet/inetmanet
} 
for the document to be downloaded in full. Parameters of video streaming for simulation purpose are appeared in Table I. All parameters are fixed except buffer size of router which is altered each time in a specific range when simulation is carried out.

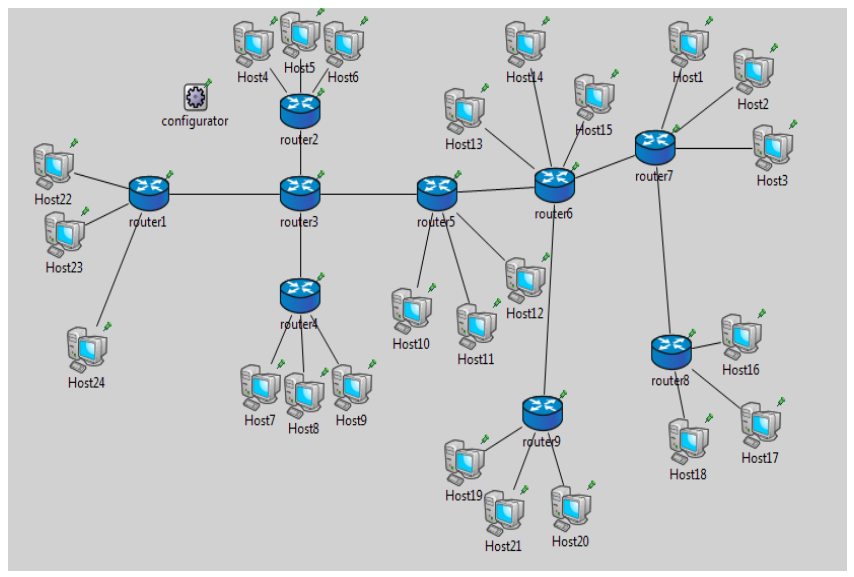

Fig. 1. Network model with point to point connection having 09 routers and 24 hosts.

Various simulations are carried out to assess the network performance in terms of mentioned indicators in Section III, utilizing the considered parameters shown in Table I. Simulations have been performed for video streaming application to examine the outcomes by varying the buffer size randomly of all intermediate range of considered routers in a specified range. That specified range is also changed for other simulations. This is called the heterogeneous buffer size approach. It should be remembered that in homogeneous approach, buffer size of all middle range considered routers is same. The created network comprises 09 routers with 24 hosts and at first middle routers are assigned different buffer sizes in a specific range to inspect the metrics which have been taken into account. Later that specific range is changed to further explore the effect of heterogeneous size of buffer on the metrics of performance. In spite of the fact that the size of buffer and range is altered, a network topology is fixed.

TABLE I. NETWORK SimUlation PARAMETERS

\begin{tabular}{|l|l|}
\hline Parameter & VALUE \\
\hline Total number of nodes & 24 (12 receiver, 12 transmitter) \\
\hline Size of Video & $1 \mathrm{~GB}$ \\
\hline Buffer size (No. of frames) & $100,75,40,20$ \\
\hline Data rate & $2 \mathrm{Mb} / \mathrm{s}$ \\
\hline Length of a packet & 1000 bytes \\
\hline Simulation time & 10000 Secs (Over all traffic time) \\
\hline Total number of routers & 9 \\
\hline Frame capacity & 4475 \\
\hline Start time & 1 Second \\
\hline Connection type & Point-to-point \\
\hline Propagation delay & $100 \mathrm{~ms}$ \\
\hline Transmit interval & 5 Seconds \\
\hline Bit Error Rate (BER) & 0.000001 \\
\hline
\end{tabular}

\section{INDICATORS OF PERFORMANCE}

To maintain the high performance of the network, many parameters of the network are analyzed. By taking up the following metrics of performance, the capability of transport protocol can be assessed for the video streaming application.

\section{A. Average Latency or End-to-End delay}

This parameter is also known as one-way delay (OWD) and portrayed as what measure of total time is required for the movement of packets from source to destination. Round-trip time in IP networks is a different term than OWD. It includes various types of delays such as processing, propagation, transmission, and queueing [8] and is not simply the half of the round-trip time. Buffers end up being speedier in light of the way that packets for transmission purpose ought to be secured in these for a significant long time and is evaluated in second [9].

\section{B. Average Throughput}

This is another important parameter to measure the performance of the network. It is defined as the aggregate payload over the whole session separated by the total amount of time. The total amount of time is ascertained by taking the distinction in timestamps between the first and last packet. Its unit is in bits per second (bps).

$$
\text { Throughput }=\frac{\text { Average Payload }(\text { Total bits transmitted })}{\text { Total Duration Observed }}
$$

\section{Buffer Size of Router}

Routers also known as packet switches have the buffers to handle the data during the time of congestion in a network which occurs due to diverse rates of transmission happen amongst packet switches and network transport. Thus, the sizing of packet switches (router) buffers is an important, pivotal and open research topic for the researchers to be addressed [3], [10].

\section{NUMERICAL RESULTS AND DISCUSSION}

The network has been built using network descriptive (NED) language in OMNET $++{ }^{1}$ and contains total 24 nodes with nodes from 13 to 24 as the sending nodes whereas 1 to 12 are the receiving nodes.

The middle routers $3,4,5,6,7$, and 9 have been considered for varying the buffer size. The application which has been considered to analyze the latency or End-to-End delay and the network throughput is the video streaming. It should be noted that for homogeneous network, the buffer size of all the midway routers is fixed whereas in the heterogeneous approach, the buffer size of all intermediate numbered routers mentioned above are randomly varied. Later, the buffer sizes are taken into account in different ranges and finally, evaluate the impact of these heterogeneous buffer sizes on the mentioned indicators, network throughput and latency for UDP performance of transport protocol.

\section{A. Interpretation of network throughput and latency or End-} to-End delay with hetrogeneous packet switch buffer sizes

The standard indicators known as network throughput and latency or End-to-End delay are the important metrics to 
analyze the performance of transport UDP protocol suite for applications like video streaming in real-time. Initially, random buffer sizes between the range of 50 to 20 has been considered and later random buffer sizes in the ranges of 30 to 20 and 20 to 10 respectively have been taken into account to examine the numerical results.

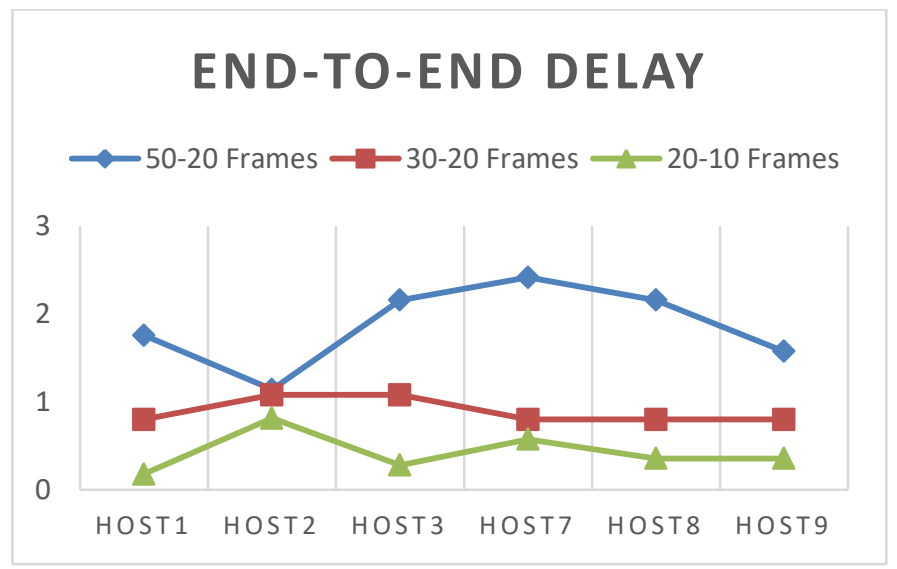

Fig. 2. Heterogeneous router buffer size vs End-to-End delay for video streaming application.

The numerical results are shown for the latency or End-toEnd delay and the network throughput in Fig. 2 and 3, respectively. It is evident from the results that latency is quite high and attains the maximum value of 2.41 seconds when the range of 50-20 has been taken as shown in Fig. 2 but at the same time, throughput is also high and gets the maximum value of 93 bits/second. Although throughput is improved in this range but this range would not be considered for video streaming application because of high latency. Further, when the buffer range has been decreased from 50-20 to 30-20 and 20-10, the latency has diminished to its minimum value of 0.18 seconds for 20-10 range but also the throughput has decreased much to its lower value of 40, especially in the range of 20-10 which is not tolerable for the video streaming application.

\section{THROUGHPUT}

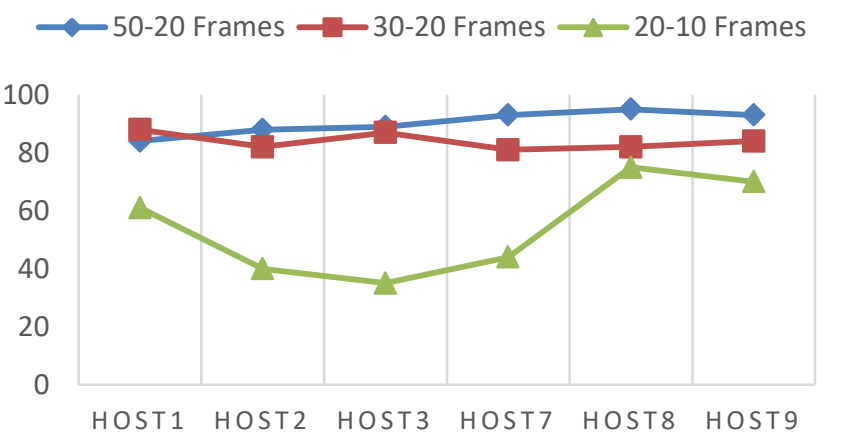

Fig. 3. Heterogeneous router buffer size vs Throughput for video streaming application.

Thus, it is obvious that with the increase in buffer size, the throughput improves but one-way delay is increasing. On the other side, if buffer size range is decreased to its lower range, delay reduces but network throughput is also declining. Thus, both high and low buffer sizes are not acceptable for the best performance of UDP protocol's video streaming application. Hence, the optimized values can be obtained when random buffer sizes are considered in the mid-range for excellent performance of UDP video streaming application where buffer size is in the range of 30-20 frames as clear from Fig. 2 and Fig. 3 results.

\section{CONCLUSION}

In this paper, heterogeneous buffer sizes in random were utilized on different routers and for different ranges to examine their effect on the performance of network for UDP protocol with video streaming application. It appeared through numerical results that packet switches heterogeneous buffer sizes as a rule influenced the general performance of the network. By thinking about bigger range of buffer sizes, throughput improved but End-to-End delay also increased which is customarily not commendable for RTC application. Be that as it may, throughput decreased on account of considering low range of buffer sizes, however, latency or delay (End-to-End) additionally diminished. In this manner, the middle of the road scope of buffer sizes range from 30 to 20 was recommended for ideal throughput and an adequate lower End-to-End delay. This work can be further extended by considering other real-time applications like voice over internet protocol (VOIP). Further, user datagram (UDP) applications maybe compared with transport control protocol (TCP).

\section{REFERENCES}

[1] Li Tang; Hui Zhang; Jun Li; Yanda Li, "End-to-End Delay Behavior in the Internet," Modeling, Analysis, and Simulation of Computer and Telecommunication Systems, 2006, MASCOTS 2006. 14th IEEE International Symposium.

[2] Damon Wischik, Nick McKeown, "Buffer sizes for core routers," ACM SIGCOMM Computer Communication, 2005, vol., no. 35(3), pp.75-78.

[3] Dhamdhere, A.; Jiang, H.; Dovrolis, C., "Buffer sizing for congested Internet links," IEEE Proceedings of INFOCOM, 2005, 24th Annual Joint Conference of the IEEE Computer and Communications Societies.

[4] Theagarajan, G.H.P., Ravichandran, S., Sivaraman, V., "An Experimental Study of Router Buffer Sizing for Mixed TCP and RealTime Traffic," ICON '06, 2006, 14th IEEE International Conference on Networks, Vol.1, pp.1-6.

[5] Ali, S.H., Nasir, S.A, Qazi, S, "Impact of router buffer size on TCP/UDP performance," Computer, Control \& Communication, IC4 2013,_3rd IEEE International Conference.

[6] Ouldooz B.K., Mahmood F., "Adaptive end-to-end QoS for multimedia over heterogeneous wireless networks," Computers \& Electrical Engineering, 2010, Vol. 36(1), pp.45-55.

[7] Masugi, M., Takuma, T., Matsuda, M., "Quality of Service assessment of video streams over IP networks based on monitoring transport and application layer processes at user clients", IEEE Proceedings of Communications, 2005, Vol. 152(3).

[8] Rohal, P., Dahiya, R., Dahiya, P., "Study and Analysis of Throughput, Delay and Packet Delivery Ratio in MANET for Topology Based Routing Protocols (AODV, DSR and DSDV)," International Journal for Advance Research in Engineering and Technology, 2013, Vol., no. 1(2), pp.54-58.

[9] Cranley, N., Perry, P., Murphy, L., "Dynamic Content-Based Adaptation of Streamed Multimedia," Journal of Network and Computer Applications, 2007, Vol. 30(3), pp. 983-1006.

[10] Prasad, R.S., Dovrolis, C., Thottan, M., "Router Buffer Sizing for TCP Traffic and the Role of the Output/Input Capacity Ratio," IEEE/ACM Transactions on Networking, 2009, Vol. 17(5) pp. 1645 - 1658. 